\title{
La encrucijada de formar a las niñas y niños como sujetos $^{1}$
}

José Carlos Flores Buenaventura²

Universidad Autónoma de México, México ${ }^{3}$

jcfbuenaventura@gmail.com

1 Artículo de investigación, producto del trabajo realizado para la tesis de Maestría en Pedagogía, UNAM.

2 Licenciado en Pedagogía por la FFyL, unam. Coordinador del Seminario de Perspectivas Críticas en Educación de México y Latinoamérica: construcción de discursos y prácticas, en la misma institución. Agradezco las correcciones y sugerencias de Horacio Cerutti, Jessica Nayeli Cruz, David Elías Hernández y a Rosario Elena Gálvez Mancilla.

3 Candidato a Magister en Pedagogía, Facultad de Filosofía y Letras, UNAM, México. 
Tema Libre

\title{
La encrucijada de formar a las niñas y niños como sujetos
}

\section{Resumen}

En este texto se presenta la tesis de que las niñas y niños se pueden formar como sujetos; consideramos que la formación de sujetos es un proceso socio-cultural y político que puede existir en el grupo social, clase, cultura o etnia de pertenencia desde que hay un ideal de cómo debe ser una niña o niño. Se realiza un análisis que parte de cuestionar la percepción dominante que se tiene sobre las niñas y los niños, que no responde a las condiciones reales de los niños mexicanos de carne hueso; en un segundo momento se revisan conceptos que permiten comprender el sentido y contenido de la categoría de sujeto en las niñas y niños; por último se presenta una propuesta de triada pedagógica para formarnos como sujetos desde la infancia: el hablar, el escuchar y el escribir.

Palabras clave: niños, niñas, infancia, sujetos, hablar, escuchar y escribir.

\section{The crossroad of forming kids as subjects}

\begin{abstract}
This article sustains the thesis that boys and girls can be formed as subject; it's considered hat the formation of subjects is a socio-cultural and political process that can exist in the social group, class, culture or ethnic group to which one belongs since there is an ideal of how children must be. An analysis is carried out based on questioning the dominant perception that is held over girls and boys, that does not answer to the real conditions of the flesh and bone Mexican children; in a second stage, the concepts that allow to understand the meaning and content of the subject category in girls and boys are reviewed; finally a proposal of a pedagogical triad to form ourselves as subjects is presented: the speaking, listening and writing.
\end{abstract}

Keywords: boys, girls, childhood, subjects, speaking, listening and writing.

\section{A encruzilhada para educar as meninas e meninos como sujeitos}

\section{Resumo}

Neste texto apresenta-se a tese de que as meninas e meninos podem ser formado como sujeitos; consideramos que a formação de sujeitos é um processo sócio-cultural e político que pode existir no grupo social, classe, cultura ou etnia de pertença e desde que há um ideal de como deve ser uma menina ou menino. Realiza-se uma análise que parte de questionar a percepção dominante que se tem sobre as meninas e os meninos, que não responde às condições reais dos meninos mexicanos de carne osso; em um segundo momento revisam-se conceitos que permitem compreender o sentido e conteúdo da categoria de sujeito nas meninas e meninos; por último apresenta-se uma proposta de triada pedagógica para formar-nos como sujeitos desde a infância: o falar, o escutar e o escrever.

Palavras-chave: meninos, meninas, infância, sujeitos, falar, escutar e escrever. 
Entre todos estos mundos subordinados, hay uno, sin embargo, que podría ser el más importante, que podría ser el único verdaderamente universal, y que constituye el punto neurálgico de todo proceso de dominación: es el mundo de los niños.

(Dorfman, 2002, p. 13)

La dominación y colonización del mundo, como bien observaba Ariel Dorfman, inicia por el mundo de los niños. Al respecto surgen diversas preguntas, tales como: ¿qué hacer para revertir o construir otros procesos que no sean los de dominación, colonización y conquista? ¿Desde dónde pararnos para poder producir otro tipo de relaciones ya sean sociales, culturales y pedagógicas que no tengan como objetivo estos procesos? ¿Cómo se forman a las niñas y niños en México a principios del siglo XXI? ¿Cómo se forma a los niños pobres y cómo a los ricos?

En este marco, este artículo surge como una necesidad de pensar de otra forma a los seres humanos que viven la infancia ${ }^{4}(2009)$, para que aquélla no siga cercada por su definición etimológica: del latín infantia, "in", como negación, y "for" hablar, significando, por lo tanto, "quien no sabe hablar" (Recuperada de http:// deconceptos.com/ciencias-naturales/infancia).

También, en este artículo se sostiene la tesis de que las niñas y niños se pueden formar como sujetos a partir de considerar la formación como un proceso sociocultural y político que puede existir en un grupo social, clase, cultura o etnia de pertenencia desde que haya un ideal de ser humano que indique, desde luego, cómo debe ser una niña o niños ${ }^{5}$.

El ser sujeto $u$ objeto no es una esencia o naturaleza del ser humano, sino una construcción socio- cultural, histórica y pedagógica. A los seres humanos que viven en esta etapa se les puede comprender como seres que son y serán, a saber, seres en movimiento.

El objetivo que se persigue con este artículo es ubicar momentos fundamentales en la formación de los seres humanos; pero no sólo en un sentido lineal y por etapas a superar, sino como procesos y experiencias que se viven para constituir al ser

4 La tesis de maestría de Shiduet Marina Castro Hernández, titulada Construcción del niño: una pedagogía corporal, permite tener una visión histórica sobre diferentes significados y usos que ha tenido la categoría de infancia a lo largo de la historia de México.

5 Se considera que el objeto de estudio de la pedagogía es la formación de los seres humanos. Este artículo se enfocará en la formación de las niñas y los niños como sujetos, por ende el objeto de estudio en este texto es la formación de los mismos como sujetos, para lo cual es necesario cuestionarse sobre la forma en que se percibe la realidad y desde donde se construye el conocimiento. 
humano durante su vida a partir del reconocimiento de que las niñas y los niños son en momentos determinados y que serán en relación con los cambios biológicos, sociales, culturales, históricos y políticos en los que viven. Del mismo modo, se busca concebirlos en la historia. Pero también como constructores de su propia historia, lo que los convierte al mismo tiempo, en objetos de estudio y en sujetos pensantes. En último término, estudiar la infancia es un proceso que se encuentra en movimiento y que no está cerrado, por lo cual se tienen que realizar acciones conscientes en una dirección u otra, lo que repercute en el futuro de las sociedades latinoamericanas o, en un sentido más amplio, en el futuro que tendrá el género humano.

\section{El problema de la formación en la infancia}

Cómo hablar y discutir de niñas y niños que quizás sólo existen en nuestra imaginación cuando la realidad de éstos se desborda entre la violencia, la pobreza y la ignorancia que hay en Latinoamérica ${ }^{6}$. A partir de este cuestionamiento, nuestra posición sobre el tema se ubica en la tensión utópica de la cual habla Horacio Cerutti: "Esa tensión entre lo deseable y lo dado que moviliza la acción y le brinda sentido a las búsquedas de algo mejor" (2009, p. 79).

En este orden de ideas, niñas y niños han sido y son seres que han realizado tareas de adultos de las peores calañas; ¿por qué no será posible que sean, desde las más tempranas edades, seres para sí, sujetos que no sólo sean hedonistas, sino responsables con su realidad, felices, justos, dignos y libres? Un posible límite teórico es pensar a la infancia a través de ideas y conceptos que no corresponden a los niños de carne y hueso que hoy día viven en la realidad mexicana. Así pues, a este conjunto de ideas, creencias, imágenes y modelos lo ubico en la metáfora del "nunca jamás"

Se puede hacer una lectura en la cual las teorías que explican el desarrollo del niño lo ubican como un ser que se desarrolla en etapas para llegar a ser adulto, pues está incompleto, carece, adolece y le falta ${ }^{7}$ siempre algo. En último término, la diferencia entre las niñas y niños y los adultos se concibe como algo negativo para los últimos. En la cotidianidad y en las teorías influyentes hay significados idealistas sobre las niñas y los niños, muchas veces con modelos cerrados, donde los conceptos de inocencia e inmadurez dan explicación de su ser.

6 Se pueden consultar con relación a la situación de la Infancia en México documentos en: Fondo de la Naciones Unidas para la Infancia (Unicef). (2010). Los derechos de la infancia y la adolescencia en México. Una agenda para el presente. México: Unicef; Cepal y Unicef. (2002). La pobreza en América Latina y el Caribe aún tiene nombre de infancia. México: Cepal y Unicef; Martínez, S. Se venden niños. México: Planeta y Sherer, J. (2013). Niños en el crimen. Barcelona: Grijalbo

7 Se puede revisar el texto de Henry Maier (2000): Tres teorías sobre el desarrollo del niño: Erikson, Piaget y Sears, en el cual se presenta un panorama general de tres perspectivas —el psicoanálisis, el constructivismo psicológico y el conductismo- que han influido en la compresión de lo que son las niñas y los niños. 
El problema no es que no se reconozca que los niños y las niñas crecen y cambian, sino que no se consideren otros factores o relaciones que los conforman en ciertos momentos, tales como su propia historicidad o las diferencias que tienen por pertenecer a diversas temporalidades y espacios. Además, hay que recordar que el crecimiento y el cambio es una constante en la vida humana.

Se observa que hay una tendencia a idealizar a la infancia. Esta idealización la sitúo como la metáfora del nunca jamás, en la cual se toma a este periodo como el mejor durante la vida y que, por esencia, es una etapa ideal en la cual no existen contradicciones en los procesos que se viven y se debería de continuar en ella. Esta metáfora esconde varios problemas. Por un lado, no se reconoce que la infancia es una construcción histórica, social y cultural —por supuesto, sin olvidar la condición biológica que se tiene-. Por otro lado, no se reconoce que la humanidad no está únicamente formada por niñas y niños, y que ellos no viven aislados, sino en convivencia con otros.

Un segundo elemento de esta metáfora es que en el "nunca jamás" las niñas y los niños no cambian y se encuentran en un mundo alterno al de los adultos, por lo cual, las decisiones de éstos no los pueden afectar y, en consecuencia, ellos pueden tomar sus propias decisiones sin mediación de algún otro actor.

Así pues, se construyen interpretaciones desde esta posición sobre la situación de los niños que no logran crear conceptos y prácticas para conocer su realidad. Por el contrario, estos discursos que alaban su inocencia o su ignorancia sobre el mundo, funcionan como una justificación para construir normas y modelos que inferiorizan y excluyen a los seres de carne y hueso, puesto que estas interpretaciones ideales no toman en cuenta su sujeción cultural, social, histórica, de género y de clase.

Lo anterior, hace parte fundamental de la infantilización de las niñas y los niños.

"Nunca jamás" es una metáfora negativa y una idealización de las condiciones reales de la infancia; una forma de exclusión y una forma de sometimiento cultural porque el modelo predeterminado se construyó sin considerar otra geografía y otra cultura. Los modelos dominantes para entender la infancia provienen tanto de países hegemónicos como de los grandes empresarios internacionales. Se habla de una infancia no existente; pero que domina en los discursos y en el sentido común de educadores y estructuras sociales en las que viven. La metáfora del "nunca jamás" remite a discursos y prácticas conducidos por un imaginario, en el cual niñas y niños están ubicados en un modelo ideal, en un país donde las variantes y las constantes de la realidad no los afectan; por lo tanto, se puede evidenciar una inmovilidad teórica en la construcción del conocimiento sobre los "infantes". 


\section{Niñas y niños como sujetos}

El preguntarse cómo formar a las niñas y niños como sujetos implica tratar de pensar de otra manera, aunque en algunos momentos se utilicen conceptos que se usan para comprender al ser humano en otras etapas de la vida como la adultez y la vejez.

A continuación se presentan algunos conceptos que pueden ayudar a repensar la categoría de sujeto en la construcción de conocimiento pedagógico sobre las niñas y los niños: a) el conocimiento de sus cuerpos, b) el conocimiento de sus propios deseos, c) la conciencia de su historia personal desde su propia interpretación, d) la ubicación de los elementos que hacen que las niñas y niños dependan de las instituciones y del momento histórico en el que viven, e) la construcción de decisiones propias y su consideración por otros grupos sociales.

\section{El conocimiento de su cuerpo}

El conocimiento del cuerpo está unido a su apropiación por parte de los individuos, ya que, como ha ocurrido en la historia de la humanidad, la pertenencia y la comprensión del cuerpo se ha ido modificando a través de los siglos. Existen diversas interpretaciones sobre éste, tales como que el cuerpo no le pertenece a la persona, sino a una divinidad superior; que los cuerpos son para cumplir ideales de los grupos dominantes en su tiempo, ya sean feudos, nobleza o gobiernos; que el cuerpo le pertenece a un individuo superior-vgr. al hombre - , como en el caso de la concepción que se ha tenido sobre el cuerpo de las mujeres; Sin embargo, en algunos momentos, ha existido, al mismo tiempo, la idea del cuerpo para sí, en potencia o en práctica, ya sea para algunos grupos o individuos.

En América Latina, la reflexión sobre el cuerpo no es nueva, pues se ha trabajado desde varios espacios. Horacio Cerutti recuerda la importancia que ha tenido este asunto para la filosofía "nuestroamericana" al señalar que: "Uno de los primeros estudiosos en Ilamar la atención acerca de la importancia del cuerpo en la filosofía nuestroamericana ha sido Arturo Rico Bovio. En dos obras decisivas para el inicio de esta discusión desarrolló la tesis central: no tenemos, sino que somos cuerpo" (2009, p. 72).

No sólo los niños pierden la propiedad de su cuerpo; también otros seres humanos, como mujeres, jóvenes, viejos, indígenas, entre otros, por lo que la reapropiación del cuerpo - ya que somos cuerpo según la tesis que recupera Horacio Cerutti de Arturo Rico Bovio - es significativa en la construcción de pedagogías pertinentes. Se habla del cuerpo de los niños porque se considera que uno de los momentos más importantes es la infancia: cuando los seres humanos pierden o construyen su pertenencia simbólica y material de su propio cuerpo. 
Ya Graciela Hierro, desde la reflexión filosófica de la educación, hacía un Ilamado a devolver el cuerpo a las niñas: "Comencemos por la infancia, que no es destino. Para ello tendremos que considerar a la mujer más allá de su biología. $Y$ en esa medida, devolver su cuerpo a la niña que generalmente le es arrebatado precisamente en la infancia" (1989, p. 99). Y si el sujeto es cuerpo, es arrebatado, muchas veces, como unidad y totalidad, puesto que se convierte en ser de otros.

Aunque esto puede pasar también cuando se es adulto como pasa en la explotación sexual y en la guerra, la apropiación del cuerpo a la que se apela en este texto no es ver a éste y otros elementos del ser humano separados, sino, como interpela el trabajo de Horacio Cerutti, darse cuenta de que el sujeto es cuerpo y, en ese sentido, se debe apropiar de él mismo así como las niñas y niños también se deben apropiar de sí mismos para ser sujetos.

La apropiación del cuerpo está íntimamente ligada a la capacidad de nombrar: nombrar las partes de su cuerpo y, por consiguiente, nombrarse a sí mismo. En la cotidianidad, existe el problema de no decir ciertas partes del cuerpo. En algunas ocasiones, esto está relacionado con los órganos sexuales, aunque en las lenguas exista un gran bagaje para nombrarlos. Por un lado, se da un sobrenombre o se utiliza lo que se considera "malas palabras"; pero la cuestión es la reducción de las palabras o el esconder por medio del silencio esto, lo cual puede interpretarse de muchas formas. Una de esas interpretaciones es que al no nombrar o no decir cuestiones sobre el cuerpo, el sujeto se esconde y se oculta y, en ese marco, se disminuye a sí mismo al percibirse en términos negativos o inferiores ${ }^{8}$.

En esta línea, un objetivo pedagógico es poder generar medios y estrategias pertinentes para que tanto niños y niñas como adultos reconozcan que son cuerpo y son seres para sí, y no para los intereses y deseos de otros.

\section{El conocimiento de sus propios deseos}

El punto en relación con el conocimiento de los propios deseos de niñas y niños, es considerar que sus deseos no son menores o inferiores a los de otros u otras. En este sentido, es primordial poder construir herramientas de mediación e interpretación de lo que dicen ellas y ellos sobre lo que sienten y desean, ya que, como ocurre con otros sujetos, es imposible sólo llevar a cabo sus deseos individuales. Así mismo, se debe buscar el espacio para construir diálogos y decisiones donde se vean incluidos.

8 Con relación al tema Michael Foucault en su texto Historia de la sexualidad. 1 La voluntad de saber, señala: "[E]l crecimiento de las perversiones no es un tema moralizador que habría obsesionado a los espíritus escrupulosos de los victorianos. Es el producto real de la interferencia de un tipo de poder sobre el cuerpo y sus placeres" (2012, p. 62). 
De este modo, para poder construir estos diálogos es necesario aprender a escuchar, como lo expresaba Carlos Lenkersdorf, donde sólo se puede escuchar a quien se puede percibir como sujeto ${ }^{9}$. Este conocer los deseos, sentimientos y emociones de los seres humanos en la infancia, como nos ha enseñado Graciela Hierro (2014), es para buscar la felicidad: la de tener múltiples formas de expresarse según la cosmovisión, tiempos y espacios donde se esté.

\section{La conciencia de su historia personal desde su propia interpretación}

En este apartado se presenta una serie de hipótesis clave para comprender que la percepción sobre la realidad que tienen los seres humanos en la infancia es diferente a la de un adulto y a la de un joven. De la misma manera, se resalta la importancia que tiene la historia personal para formar los momentos y experiencias significativas que permiten que el sujeto construya una identidad y una memoria social e histórica.

La primera hipótesis es que las niñas y niños construyen una interpretación propia de su historia. La segunda hipótesis es que los seres humanos constituyen su memoria histórica desde el nacimiento, por lo cual, no se puede decir, en ningún momento a lo largo de la vida, que no se tenga memoria; más bien, se debe discutir qué tipo de memoria social e histórica tiene cada individuo como, por ejemplo la memoria de su pueblo o, como afirmaba Paulo Freire (2005), la memoria del opresor - aunque recordemos que Freire hablaba en su libro la Pedagogía del oprimido de la conciencia-. Así pues, se puede decir que un elemento fundamental para que haya conciencia es la memoria.

Una tercera hipótesis, que va de la mano con las dos anteriores, es que durante la infancia, los seres humanos perciben diferente el tiempo o que viven diversas temporalidades a las que se viven en la edad adulta o en la vejez ${ }^{10}$.Dichas interpretaciones y percepciones dependen de la cultura y del momento histórico en el cual se vive, como demuestra en sus investigaciones Carlos Lenkersdorf (1996), donde señaló que al pertenecer a diferentes culturas y familias lingüísticas, los seres humanos han logrado construir diferentes cosmovisiones. Del mismo modo, se puede decir que éstos se apropian de esas múltiples formas de concebir el mundo durante la infancia. Por ende, es primordial considerar el elemento histórico y cultural en la formación de la misma. Al hablar de una interpretación propia de la historia de niños y niñas, ésta responde a los grupos o sociedades a los cuales pertenecen, pues aquéllos no rehazan todo el cosmos, sino que lo interiorizan y lo reactualizan a través de sus procesos psíquicos y sus acciones diarias y cotidianas.

9 Carlos Lenkersdorf en Aprender a escuchar (2008) señala que: "'ab'i) Tanto en el sentido de escuchar como de sentir, quiere decir, percibir palabras y sentimientos desde la perspectiva del otro o de los otros" (p.122).

10 Un estudio fundamental para la comprensión del tiempo se encuentra el libro de Guadalupe Valencia, titulado Entre Cronos y Kairós. Las formas del tiempo socio histórico (2007). 


\section{Ubicación de los elementos que hacen que las niñas y niños dependan de diferentes instituciones y del momento histórico en el que viven}

Este elemento, con referencia a la dependencia de los niños a diferentes instituciones y sujetos, es un tema a debatir más a fondo, lo cual es necesario hacerlo y este apartado sólo es una introducción al tema y al problema.

Ante lo dicho previamente, aparece un factor importante: la dependencia económica y emocional que ellas y ellos tienen hacia los adultos y a diferentes instituciones sociales. En esta población es un factor muy visible y, además, es un referente en la cotidianidad; pero también parecería ser un referente negativo en el cual las niñas y niños pierden la capacidad de decisión y de intervención en diferentes espacios sociales.

Esta característica que ha servido para identificar a niñas y niños es lo que se denota como "ser menor de edad", ser dependiente y ser no- ciudadano. Es una metáfora negativa constante que se utiliza para recordar la dependencia de los "infantes" a los adultos que, por diversas razones, no les permite ser autónomos económica ni emocionalmente. Si se lleva esta metáfora a otro nivel, el "ser menor de edad", en relación con la dependencia económica, también aplica para ciertos países y regiones geográficas. Ya Ariel Dorfman había reflexionado sobre la infantilización de las naciones en su libro Patos, elefantes y héroes. La infancia como subdesarrollo. Ahí señala que:

Y como aquellas capas, comunidades, razas, continentes, individuos, clases, que no caben en el orden oficial, tienden a ser mirados como "niños", como seres incompletos que aún no han arribado a una edad madura, es la literatura infantil o la infantilización de la literatura masiva adulta, la que conforma la base de este proceso. Ya lo dijo el niño mimado (el Wunder Kind) de la política internacional Henry Kissinger, cuando justificaba la intervención de sus servicios secretos en el derrocamiento del presidente legítimo de mi país (Chile): "No veo por qué debemos quedarnos sin hacer nada mientras miramos cómo un país se convierte al comunismo debido a la irresponsabilidad de su propio pueblo (2002, p. 14).

Una perspectiva que permite reflexionar sobre la dependencia desde otro lugar es la cuestión del cumplimiento de los derechos humanos, los cuales, como se recuerda en una de sus caracterizaciones, son las mínimas condiciones que deben tener las personas para vivir con dignidad; por ejemplo, tener alimentación, educación, salud, vivienda y una larga lista de derechos que se han ganado en luchas históricas en diversas partes del mundo. En esos términos, el depender de otros no implica convertirse en objetos de éstos, sino que deberían de cumplirse sus derechos humanos, sin rebajar su calidad de sujeto. 


\section{Construcción de decisiones propias y ser tomados en cuenta por otros grupos sociales}

Elaborar estrategias para que niñas y niños puedan construir sus propias decisiones es un objetivo pedagógico y político, ya que se tendrían que constituir mecanismos democráticos en los cuales participen en la casa, en la familia, en el club deportivo y en todos los espacios donde ellas y ellos viven. En última instancia, se trata de concebir que niñas y niños tengan, desde sus muchos o pocos referentes, una ubicación de sí mismos en la realidad social e histórica en la que viven y una ubicación en la familia, en la escuela, entre sus pares y los múltiples espacios y grupos sociales a los cuales pertenecen. Así mismo, se busca reconocer que tienen la capacidad de tomar decisiones y que constantemente se forman en la posibilidad de lograr diálogos o en la imposibilidad de llegar a ellos. El diálogo es fundamental para el intercambio de intereses y deseos para la búsqueda de la construcción de decisiones colectivas y sociales.

\section{La infancia: el hablar, el escuchar y el escribir}

A continuación se presenta una propuesta basada en una tríada de acciones pedagógicas en la formación de la infancia en México. Esa tríada se compone del "bien hablar", del "saber escuchar" y del "saber escribir" para buscar las posibilidades de construir diálogos que conduzcan a praxis que transformen la realidad hiriente en la que vivimos. De la misma manera, esta tríada tiene un sentido democrático, en el cual no sólo sean unos cuantos, sino "muchos" los que tengan acceso a espacios sociales, políticos, científicos, artísticos y cotidianos en donde se enseñan estas habilidades que se consideran fundamentales.

El hablar "bien" ha sido esencial para la participación de los ciudadanos, pues es la disciplina de la oratoria el mecanismo de la memoria histórica en la cual se registran los diferentes saberes y conocimientos producidos en una época sobre el tema ${ }^{11}$. Así mismo, la oratoria es importante porque ha sido considerada como la palabra que puede transmitir ideas, plantear proyectos y producir acciones concretas ${ }^{12}$. Se apela a esta disciplina milenaria para que se le enseñen a las niñas y los niños herramientas para poderse expresar y trabajar internamente en el reconocimiento y expresión de deseos y emociones. En último término, se considera que es pertinente plantear una actualización de la oratoria para que sea viable su enseñanza en un sistema educativo público y poder elaborar, con los especialistas y los actuales oradores en México, lo que se podría llamar "oratoria para niñas y niños en México".

11 Este tema se puede ver en: Cota, J. (1985). El hombre es su palabra (variaciones en torno a la oratoria), México: Consejo Nacional de Recursos para la atención de la juventud.

12 En relación a este tema le agradezco a Francisco Javier Correa López. 
El hablar no está desvinculado del escuchar como ha enseñado Carlos Lenkersdorf en su libro Aprender a escuchar (2008). Escuchar no es sólo percibir ruidos, sino lograr percibir el mensaje que otro dice para que se logre la comunicación. Esto sólo es posible, argumentaba Lenkersdorf, entre sujetos.

Ahora bien, el concepto de "hablar bien" ha sido polémico. Por dicha razón, este concepto se utiliza no desde una visión monista o de un sentido común dominante, sino a partir de una visión que toma en cuenta la gran diversidad cultural que existe no sólo en América Latina, sino en el mundo entero. Por ello, cada lengua y cultura tiene sus propios conceptos para determinar qué es hablar bien desde sus principios gramaticales, semánticos y sintácticos. Esto es importante subrayar, ya que cuando se habla de lo correcto o de lo incorrecto, bien parece que se regresa a los términos de dominación y domesticación que han existido en la historia de América Latina. Desde luego, no es esa la intención, más bien se busca hacer presente la pluralidad que existe en el mundo en relación con "hablar bien" y "aprender a escuchar".

El tercer elemento de esta tríada es el "saber escribir" que es una forma de presentar en forma gráfica lo que piensan, sienten y viven los seres humanos en sus múltiples relaciones con otros seres y con la realidad en la cual viven ${ }^{13}$. El objetivo no es sólo que se supere el problema del analfabetismo funcional que afecta a la mayoría de la población que viven en clases medias, bajas y pobres, sino aspirar a que se formen desde las más tempranas edades a los seres humanos como escritores, lo cual no implica que un escritor sea solamente aquél que publica libros en el mercado editorial, sino también aquél que pueda producir la palabra escrita y repercutir en la construcción y transformación de la realidad en donde se vive ${ }^{14}$.

El "hablar bien", el "aprender escuchar" y "escribir" son prácticas culturales que, desde una perspectiva pedagógica, se consideran como estrategias para que las niñas y los niños se formen como sujetos.

13 Se considera pertinente que para llevar a cabo esta tarea pedagógica se puede hacer una lectura sobre la obra de Paulo Freire que hoy tiene mucho que decir para poder construir estrategias, acciones y procesos pedagógicos que permitan que las niñas y los niños posean la palabra tanto oral como escrita. Entre algunos de sus textos se encuentran: La educación como práctica de la libertad (1989), Pedagogía del oprimido (1970) y Por una pedagogía de la pregunta (2010).

14 Paulo Freire (2005) señala que: "Está búsqueda nos lleva a sorprender en ella dos dimensiones acción y reflexión —en tal forma solidarias, y en una interacción tan radical que, sacrificada, aunque en parte, una de ellas, se resiente inmediatamente la otra. No hay palabra verdadera que no sea una unión inquebrantable entre acción. De ahí que decir la palabra verdadera sea transformar el mundo" (p. 106). 


\section{Conclusiones}

Este artículo presentó reflexiones que permiten pensar y construir prácticas para formar y percibir a los seres humanos como sujetos desde la infancia, lo cual no implica concebir que ser sujeto sea una esencia o cuestión natural, sino más bien es una construcción histórica y, por tanto, pedagógica que siempre está en movimiento: en diferentes circunstancias y momentos de la vida se puede ser "sujeto" u "objeto".

De la misma manera, se presentó a lo largo del texto conceptos que se consideran importantes para poder utilizar la categoría de sujeto en las niñas y en los niños, los cuales son: a) el conocimiento de sus cuerpos, b) el conocimiento de sus propios deseos, c) la conciencia de su historia personal desde su propia interpretación, d) la ubicación de los elementos que hacen que las niñas y niños dependan de las instituciones y del momento histórico en el que viven, y e) la construcción de decisiones propias y ser tomados en cuenta por otros grupos sociales. Hay que tener en cuenta también que el percibirlos como objetos o sujetos repercute en los proyectos educativos que se llevan a cabo o que se realizaran en el futuro, y por ende en los proyectos de sociedad en Latinoamérica.

En otro momento se esbozó brevemente una propuesta formativa que está integrada por una tríada pedagógica: el "bien hablar", el "aprender escuchar" y el "aprender a escribir" que se consideran fundamentales para poder formar sujetos.

Para concluir este artículo apeló a la desinfantilización de la infancia y la pedagogía, ya que se considera que el conocimiento pedagógico es necesario para la construcción de proyectos sociales para la liberación y emancipación de los pueblos y sociedades. Es necesario subrayar, dentro de los límites de la pedagogía, el objetivo de la desinfantilización de las niñas y los niños, pues este proceso permite dejar de percibir a éstos como objetos, como seres inferiores y formarlos como sujetos en el reconocimiento de su dignidad humana. El momento actual demanda formar sujetos desde la infancia como una manera de resistir a la colonización y explotación de nuestros países latinoamericanos por parte de otros países para así lograr los objetivos de independencia, democracia, libertad y justicia que se necesitan en nuestra América. 


\section{Referencias}

Castro, S. (2009). Construcción del niño: una pedagogía de lo corporal (tesis de maestría). unAM, México D.F, México.

Cepal y Unicef. (2002). La pobreza en América Latina y el Caribe aún tiene nombre de infancia. México: Cepal y Unicef.

Cerutti, H. (2009). Lo utópico operante en la historia (utopía, praxis de la resistencia en nuestra América). En Agora Philosophia, 8(19-20), pp.77-88.

Cerutti, H. "Preliminares hacia una recuperación del cuerpo en el pensamiento latinoamericano contemporáneo". En: N. Durán y M. Jiménez (Coord.). Cuerpo, sujeto e identidad (pp.72-). México: ISSUE-UnAm y Plaza Valdés Editores.

Cota, J. (1985). El hombre es su palabra (variaciones en torno a la oratoria), México: Consejo Nacional de Recursos para la atención de la juventud.

Fondo de la Naciones Unidas para la Infancia (Unicef). (2010). Los derechos de la infancia y la adolescencia en México. Una agenda para el presente. México: Unicef.

Foucault, M. (2007). Historia de la sexualidad. 1 La voluntad de saber. México: Siglo XXI editores.

Freire, P. (2005). Pedagogía del oprimido. México: Siglo XXI.

Hierro, G. (1989). De la domesticación a la educación de las mexicanas. México: Fuego Nuevo.

Hierro, G. (2014). La ética del placer. México: Diversa.

Lenkersdorf, C. (1996). Los hombres verdaderos. Voces y testimonios tojolabales. México: Siglo XXI.

Lenkersdorf, C. (2008). Aprender a escuchar. México: Plaza y Valdes.

Maier, H. (2000). Tres teorías sobre el desarrollo del niño: Erikson, Piaget y Sears. Barcelona: Paidos.

Martínez, S. (2009). Se venden niños. Barcelona: Planeta.

Sherer, J.(2013). Niños en el crimen. Barcelona: Grijalbo.

Valencia, G. (2007). Entre Cronos y Kairós. Las formas del tiempo sociohistórico España: Antropos-UNAM.

Recibido: 30 de enero 2016

Aceptado: 20 marzo 2016

Cómo citar: Flores, J. (2016). La encrucijada de formar a las niñas y

niños como sujetos. Praxis Pedagógica, 19, 29-41 